\title{
DIAGNOSTIC DIAGRAMS FOR PLANETARY NEBULAE AND SYMBIOTIC STARS
}

\author{
A. GUTIERREZ-MORENO, H. MORENO and G. CORTES \\ Departamento de Astronomía, Universidad de Chile, Casilla 36-D, Chile
}

AbSTHACT. One of us (Gutiérrez-Moreno 1988) presented some time ago a preliminary version of two diagrams for separating planetary nejulae (Piv) from symbiotic stars (SS); these aiagrans are based on the strength of [O III] lines $\lambda \lambda 4363,5007$. Now a new version is presented, which includes more objects and uses measured total intensities corrected by reddening.

It is confirmed that the diagrams separate very well. PN from SS. Besides, it is found that they also separate fairly well $\mathrm{S}$ - and D-type SS, with a certain overlap between the regions occupied by both kinds of objects. The diagrams also separate very young Piv, which occupy in the diagram an intermediate region between SS and PN.

It is found that D-type SS with PN-like spectra for which we have intensities measured during a sufficiently large time interval seem to move down in the $\mathrm{F}(4363) / \mathrm{F}(\mathrm{H \gamma})-\mathrm{F}(5007) / \mathrm{F}(\mathrm{H \alpha})$ diagram, thus suggesting an evolution from the SS region to the PN region. The behaviour of two very young PN, IC 4997 and Ho 12, for which we have found observations made about 50 years ago, seem to confirm this suggestion, since in their first observed position, they were located in the D-type SS region. An interesting object is V1016 Cyg, corsidered probably as a PN being formed. This objects is classified as a D-type SS, which suffered an eruption in 1965. From then on it started moving upwards in the diagram, arriving to a maximum $I(4363) / H(\gamma)$ value in 1974 , when it began its downward motion until the 1937 observations, when it occupied again practically the same position than in 1965.

REFEREINCES

Gutiérrez-Moreno, A. 1988, in "Progress and Opportunities in Southern Hemisphere Optical Astronomy". CTiO 25th Anniversary Symposium", eas. V.M. Blanco and M.M. Phillips (Utan: Brigham Young University), A.S.P. Conference Series, 1, 12. 